\title{
Integralgleichungen für einige Randwertprobleme für Gebiete mit
} Ecken

\section{Doctoral Thesis}

Author(s):

Arbenz, Kurt

Publication date:

1958

Permanent link:

https://doi.org/10.3929/ethz-a-000090099

Rights / license:

In Copyright - Non-Commercial Use Permitted 
Prom. Nr. 2777

\title{
Integralgleichungen für einige Randwertprobleme für Gebiete mit Ecken
}

\author{
Von der \\ EIDGENOSSISCHEN TECHNISCHEN \\ HOCHSCHULE IN ZÜRICH \\ zur Erlangung \\ der Würde eines Doktors der \\ Mathematik \\ genehmigte \\ PROMOTIONSARBEIT
}

vorgelegt von
KURT ARBENZ
von Winterthur und Gross-Andelfingen $\mathrm{ZH}$

Referent: Herr Prof. Dr. E. Stiefel

Korreferent: Herr Prof. Dr. A. PFluger

1958 Zürich Buchdruckerei Berichthaus 


\section{Einleitung}

Ein häufiges Problem, das sich in den verschiedensten Zusammenhängen stellt, ist die konforme Abbildung eines einfach zusammenhängenden Gebietes mit Ecken auf den Einheitskreis. Obwohl durch den RiemanNschen Abbildungssatz die Existenz der Abbildung gesichert ist, kann die Abbildung in den wenigsten Fällen explizite angegeben werden.

Ist die Abbildungsfunktion $f(z)$ auf dem Rand des Gebietes $G$ bekannt, so ist nach der CAUCHYschen Integralformel die Funktion auch in $G$ bestimmt. Es genügtdaher, den Polarwinkel $\sigma$ der Bildpunkte der Randpunkte zu bestimmen. Für $\sigma$ gilt nun die bekannte LichtensteIn-Gerschgorinsche Integralgleichung [4]. Diese Fredholmsche Integralgleichung gilt in ihrer gewöhnlichen Formulierung nicht für Gebiete mit Ecken und hat ausserdem den Nachteil, dass die Lösung nicht eindeutig ist. Von Strefel [3] wurde eine Integralgleichung aufgestellt, die den letzteren Nachteil behebt, aber auch nur für Gebiete mit stetig gekrümmter Randkurve gilt. Weitere Modifikationen der Gerschgorinschen Gleichung haben Birkhoff, Young und Zarantonello zitiert [7].

Es ist nun das Ziel dieser Arbeit, für $\sigma$ eine eindeutig lösbare Integralgleichung anzugeben, die für Gebiete mit Ecken gültig ist und trotzdem numerisch bequem ausgewertet werden kann.

Dabei stützen wit uns auf die Theorie von RADON [2] über die Integralgleichungen der Potentialtheorie für Gebiete mit Ecken, die teilweise unter Benutzung funktionalanalytischer Methoden für unsere Zwecke neu aufgestellt wird. Mit Hilfe dieser Grundlagen werden dann die Eigenwerte der auftretenden Integraloperatoren untersucht. Die bekannten Resultate für Gebiete mit stetig gekrümmter Randkurve [5] gelten auch noch etwas eingeschränkt für Gebiete mit Ecken. In beweistechnischer Hinsicht ergeben sich wegen der Verwendung des StIELtjesintegralbegriffes einige Schwierigkeiten. Zur Demonstration der Wirksamkeit dieser neuen Methode wird das Quadrat konform auf den Einheitskreis abgebildet. Die erreichte Genauigkeit ist trotz dem bescheidenen Rechenaufwand verblüffend.

Im zweiten Teil wird versucht, die Integralgleichungsmethode zur Lösung von Randwertaufgaben auf das Plattenproblem anzuwenden. Die Resultate sind eher bescheiden. Es gelingt nicht, irgendwelche Aussagen über die Lage der Eigenwerte zu machen, da die GreENsche Formel, die für Potentialprobleme ein wesentliches Hilfsmittel darstellt, nicht angewendet werden kann. Ausserdem können gemischte Randwertprobleme nicht gelöst werden, da die Kerne in den auftretenden Integraloperatoren nicht beschränkt sind. Trotzdem wird noch ein Plattenproblem durchgerechnet, um zu zeigen, dass die Methode für spezielle Randwertprobleme gute numerische Resultate liefert.

Es bleibt mir noch die schöne Aufgabe zu danken: Herrn Professor E. Striffel für sein stetes Interesse und seine wertvollen Anregungen sowie Herrn Professor A. PFLuger für seine Kritik und Verbesserungsvorschläge. 